\title{
What is Industrial Engineering (IE)?
}

\section{Andy TC Wong*}

Department of Manufacturing Engineering and Engineering Management, City University of Hong Kong, China

In essence, IE can be viewed as a collection of engineering approaches to examine any complex systems or processes. As a result, possible solutions or strategies can be proposed and applied to fix the problems or improvements. In either case, two major engineering approaches can be adopted: mathematical formulations and quantitative methods. In general, a typical process can be defined as a procedure of transforming raw input into output which is more useful or valuable to the real world. By integrating more than one process, a system can be formed. In the study of IE, there are some common issues that need to be addressed. For example: what inputs are critical for a process to generate desirable outputs? What are the nature and strength of association between inputs? What is the effectiveness or efficiency of a process? What are the nature and strength of associations between several processes? Which process is most influential to the system? What is the system performance and how can it be measured? Answers for the questions above would be determined using a variety of mathematical and quantitative tools, or known as IE tools.

\section{How is it relevant to Engineering Management (EM)?}

Management can be described as process of manipulating all available resources to accomplish a goal under some constraints. If IE tools or some other scientific methods are employed to the field of management, this can be termed Engineering Management (EM). As we know, management issues mostly are related to human behavior which is hardly to be quantified, hence, measured exactly. So, how IE tools can be successfully used to solve managerial problems or make managerial improvements? First of all, data or information is important in order to mathematically formulate (describe) the background of a managerial issue. Secondly, we need to identify the problems (the gap between the current status and the expected status) or improvements (the opportunity to achieve a more desirable status). In this regard, IE tools can be applied to define the gap or the opportunity. Thirdly, we need to consider and evaluate all possible solutions or strategies such that the best solution or strategy can be identified. Next, an implementation plan needs to be formulated to specify how the best solution or strategy can be used and what are the possible resistances and challenges. Lastly, we need to monitor the system status after implementation such that corrective action can be taken in case of any deviations. With the use of IE tools, subjective evaluation and judgment would be possibly minimized. Thus, more attention is given to the facts or truths which are more evidence-oriented. However, in some cases, empirical knowledge is required if an issue is completely new or no past data/information can be found.

In short, IE knowledge (tools and concepts) is useful to minimize the level of subjectivity when resolving a managerial issue. At the same time, more factual evidence can be utilized to support the decisionmaking process. However, IE is not a panacea. Since human will evolve, new and more complex managerial issues will be observed. That's why a key concept of IE to make continuous improvement is useful to management. In other words, the problem-solving and decisionmaking processes are essentially life-long, and there is no specific end point or finish line. Theoretically speaking, there will be an end once perfection is achieved. But, if we try to look for the word "perfection" in the IE dictionary, it will never be there.
*Corresponding author: Andy TC Wong, Department of Manufacturing Engineering and Engineering Management, City University of Hong Kong, China, E-mail: tcwong@cityu.edu.hk

Received November 05, 2012; Accepted November 08, 2012; Published November 12, 2012

Citation: Wong ATC (2012) What is Industrial Engineering (IE)? Adv Robot Autom S5:e001. doi:10.4172/2168-9695.S5-e001

Copyright: (c) 2012 Wong ATC. This is an open-access article distributed unde the terms of the Creative Commons Attribution License, which permits unrestricted use, distribution, and reproduction in any medium, provided the original author and source are credited. 